\title{
Home-Based Walking Program Increases Leg Muscle Strength in Hemodialysis Patients
}

\author{
Orawan Ponngeon, PhD candidate, PT ${ }^{1)}$, Rungchai Chaunchaiyakul, PhD, $\mathrm{PT}^{2}$, \\ KRIENGSAK VAREESANGTHIP, PhD, MD ${ }^{3)}$, DUSIT LUMLERTGUL, MD ${ }^{4}$, \\ SumaleE NAKUM, BSc, NU ${ }^{5)}$, WATTANA JALAYONDEJA, PhD, $\mathrm{PT}^{1)}$ \\ 1) Faculty of Physical Therapy, Mahidol University: 999 Phuttamonthon 4 Road Salaya, Nakhon Pathom \\ 73170, Thailand.TEL: +66 2-441-5450,FAX: +66 2-441-5454,E-mail: ptwjl@mahidol.ac.th \\ ${ }^{2)}$ College of Sports Science and Technology, Mahidol University \\ ${ }^{3)}$ Faculty of Medicine Siriraj Hospital, Mahidol University \\ 4) Faculty of Medicine, Chiang Mai University \\ ${ }^{5)}$ Faculty of Medicine Maharaj Nakorn Chiang Mai Hospital
}

\begin{abstract}
Purpose] To determine the effects of a home-based walking program on lower extremity muscle strength of hemodialysis patients. [Methods] Twenty-six hemodialysis patients were divided into a training and a control group. Lower extremity muscle strength was measured using a back and leg dynamometer prior to and at the 4th, 8th, and 12th weeks of the study period in both groups. The training group performed the home-based walking program, an individually prescribed walking program which progressively increased in duration by incrementing the number of steps by $10 \%$ every two weeks. Subjects in the control group were asked to keep to their routine activities, as much as possible, during the study period. [Results] Lower extremity muscle strength in the training group remarkably increased from $52 \mathrm{~kg}$ at baseline to $68 \mathrm{~kg}$ at the 12 th week ( 4 th week $=58 \mathrm{~kg}$, 8th week $=63 \mathrm{~kg}$ ), whereas no change in the control group was found (baseline $=51 \mathrm{~kg}$, 4 th week $=52 \mathrm{~kg}$, 8 th week $=51 \mathrm{~kg}, 12$ th week $=48 \mathrm{~kg}$ ). [Conclusion] A homebased walking exercise program improved lower extremity muscle strength of hemodialysis patients. Patients who have stable medical conditions should be encouraged to participate in this home-based walking exercise program to improve their abilities to perform activities of daily living.

Key words: Hemodialysis, Walking, Muscle strength
\end{abstract}

(This article was submitted Sep. 29, 2010, and was accepted Nov. 5, 2010)

\section{INTRODUCTION}

Dialysis patients have limited exercise ability and poor physical function when compared to the general population ${ }^{1)}$. Impaired physical function has been associated with low quality of life and high mortality rate in this population ${ }^{2,3}$. The causes of poor physical function have not been fully elucidated, but it is likely that muscle abnormalities contribute to abnormalities in both structure and function of this problem ${ }^{4}$. Exercise training has been conducted for hemodialysis patients. Aerobic exercise, strengthening exercise and the combination of both aerobic and strengthening exercises are used as exercise tools for this specific group of patients ${ }^{5-7}$. However, these exercises occasionally need sophisticated instruments and can be time-consuming when patients and care givers have to devote their time to travel to a rehabilitation center. The common barriers to participation in these exercises are transportation problems to the rehabilitation center and the patients' need to stay home on non-dialysis days. Homebased exercise on non-dialysis days has been considered as an alternative rehabilitation tool for hemodialysis patients who cannot travel to a rehabilitation center ${ }^{8}$.

Walking is the most frequently adopted type of regular physical activity and is usually performed during recreational activities, transportation, occupational tasks and activities of daily living by the general population ${ }^{9}$. Walking can be used as a low-impact exercise program that improves functional performance ${ }^{10)}$. Recently a training program based on walking at increasing speed was proposed for patients on hemodialysis ${ }^{11)}$. However, this exercise protocol requires a specific, stepwise prescription. Although it is tempting to adopt the prescription of 10,000 steps/day, this number of walking steps is a high and unrealistic goal for typical sedentary free-living populations or chronic disease patients ${ }^{12-15)}$. A previous study suggested that selfreported goal setting based on baseline values and feedback is a more realistic approach to increasing the activity of individuals ${ }^{16)}$. The purpose of this study was to investigate the effects of a home-based walking exercise for hemodialysis patients on lower extremity muscle strength. 


\section{SUBJECTS AND METHODS}

This study had a single-blind study design. Twenty-six end-stage renal failure disease patients who were being treated with hemodialysis participated in this study. Patients were divided into training $(\mathrm{n}=13)$ and control groups $(n=13)$, and the experimental protocol was clearly explained to the patients. Patients then signed informed consent forms before the experiment began. The study was approved by the Ethics Committee of the Faculty of Associated Medical Sciences, Chiang Mai University, Thailand, and complied with the Declaration of Helsinki. Inclusion criteria for the patients were: hemodialysis treatment $2-3$ times/week, stable medical status (hemoglobin $\geq 10 \mathrm{~g} / \mathrm{dl}$, Kt/Vurea $>1.2$ ), unchanged medication and independent ambulation. Patients who had myocardial infarctions within 6 weeks prior to or during training, uncontrolled hypertension, uncontrolled arrhythmia, unstable angina, any neuromusculoskeletal problems or cardiorespiratory complications which might have affected testing or the training program were excluded. The subject characteristics, age, height and weight, renal-related data and the comorbidity-age index are presented in Table 1. The data shows that patients had similar ages, heights, weights and renal-related data.

Muscle strength refers to the maximal force that can be generated by a specific muscle or muscle group. Isometric or static strength, with constant muscle length during muscle activation, can be measured conveniently with a variety of devices including cable tensiometers and dynamometers. The dynamometer is a relatively cheap and portable instrument that can be used outside of the laboratory ${ }^{17)}$. Lower extremity muscle strength in this study was evaluated by using a back and leg dynamometer (TKK 5402 BACK-D, TAKEI Scientific Instruments Lo, Ltd., Japan). This device measures strength at one specific point in the range of motion and has high test-retest reliability $(\mathrm{r}=0.80)^{17,18)}$. Lower extremity muscle strength was recorded in a standing position while both knees were bent at an angle of $135^{\circ}$. The patients' hands held a handle bar which was placed on the thighs. Chain length was adjusted according to the above position. Using pronated grips, patients were asked to slowly straighten their legs up to their maximal level without using back or shoulder muscles. Every subject received consistent encouragement until the experimenters read no further changes on the scale. The test was performed 3 times with 1-min rest intervals; the highest value was selected and recorded ${ }^{17,19)}$.

To quantify walking ability, the training group was instructed to wear a pedometer (New-Lifestyles NS-800, New-Lifestyles, Inc., Japan) to monitor their average number of daily steps. This was done a week prior to participation in the exercise program. Each individual's training program was designed according to the average of number of daily steps on non-dialysis days, which was recorded as that individual's baseline step count. A progressive exercise program was designed by gradually increasing the number of steps by $10 \%$ every two weeks. Patients walked until the target number of steps was reached for each non-dialysis day. They did not need to do all of the walking in one contiguous block, but were allowed to accumulate their steps throughout the day. The patients were asked to return their pedometers to the investigator by the end of the week in order to monitor their adherence to the walking program. The walking exercise program continued for twelve weeks and was performed at least three times a week. Each patient kept a daily log book. The control group was asked to keep to their routine daily physical activities during the study period. A two-way $(2 \times$ 4) mixed analysis of variance was used to compare the effect of the walking program on lower extremity muscle strength. The independent variables included one between group variable (training and control) and one within group variable, time (before and after the 4th, 8 th, and 12th weeks of walking program). The Bonferroni correction was used for post hoc analysis in the case of a significant main effect

Table 1. Characteristics of Subjects

\begin{tabular}{lcc}
\hline & Control group & Training group \\
\hline Gender, M/F & $8 / 5$ & $9 / 4$ \\
Age, years & $53 \pm 5$ & $50 \pm 5$ \\
Height, cm & $159 \pm 6$ & $164 \pm 8$ \\
Weight, kg & $54.0 \pm 8.9$ & $57.9 \pm 9.2$ \\
BMI, kg/m2 & $21.2 \pm 3.0$ & $21.7 \pm 3.4$ \\
Time of HD, months & $34 \pm 22$ & $50 \pm 38$ \\
& (Range $10-74)$ & (Range $22-147$ ) \\
Frequency of HD, times/week & 3 times/week $=9$, & 3 times/week $=9$, \\
Kt/V & 2 times/week $=4$ & 2 times/week $=4$ \\
Hb, g/dL & $1.6 \pm 0.3$ & $1.6 \pm 0.3$ \\
UF remove, mL & $11.0 \pm 1.0$ & $11.1 \pm 0.9$ \\
Comorbidity-age index, $\mathrm{n}$ & $2577 \pm 1336$ & $2846 \pm 987$ \\
$\quad$ Low & & 11 \\
Medium & 9 & 2 \\
$\quad$ DM & 4 & 2 \\
\hline
\end{tabular}

Values are means $\pm \mathrm{SD}$ of the data. $\mathrm{M}=$ male; $\mathrm{F}=$ female; $\mathrm{BMI}=$ body mass index; $\mathrm{HD}=$ hemodialysis; $\mathrm{Kt} / \mathrm{V}=$ dialysis dose $\mathrm{Hb}=$ hemoglobin concentration; $\mathrm{UF}=$ ultrafiltration; $\mathrm{DM}=$ diabetes mellitus. 
difference between groups. The difference was considered significant when alpha $<0.05$.

\section{RESULTS}

The average numbers of steps on dialysis and nondialysis days prior to exercise in the training groups were 4,067 $\pm 1,923$ and 5,834 $\pm 2,176$ (mean $\pm \mathrm{SD}$ ) steps/day, respectively. After the completion of the 12-week exercise program, the average numbers of steps on dialysis and nondialysis days were 7,968 $\pm 5,956$ and 13,284 $\pm 5,068$ steps/ day, respectively. The percent changes of the numbers of steps on dialysis and non-dialysis days were $206.84 \pm$ $132.91 \%$ and $237.82 \pm 75.34 \%$, respectively (Table 2 ).

The lower extremity muscle strength in the training group increased from $52 \pm 5 \mathrm{~kg}$ at baseline to $68 \pm 4 \mathrm{~kg}$ (mean \pm $\mathrm{SE}$ ) after completion of the walking program (4th week $=58$ $\pm 4 \mathrm{~kg}$, 8th week $=63 \pm 4 \mathrm{~kg}$ ), whereas there was no statistically significant difference in the control group over time (baseline $=51 \pm 5 \mathrm{~kg}$, 4th week $=52 \pm 5 \mathrm{~kg}$, 8th week $=51 \pm 5 \mathrm{~kg}, 12$ th week $=48 \pm 4 \mathrm{~kg}$ ). There was a main effect of time, $\mathrm{F}(3,72)=3.591, \mathrm{MSE}=220.674, \mathrm{p}=0.018$. There was also a significant interaction between group and time, $\mathrm{F}$ $(3,72)=7.353, \operatorname{MSE}=451.835, \mathrm{p}<0.001$. This means the effects of the walking program on training and control groups was different at different times. Post hoc analysis using the Bonferroni correction indicated that the lower extremity muscle strength of the training group increased after the 8th and 12th weeks of training from baseline. However, there was no statistically significant difference in lower extremity muscle strength in the control group over time. There was no main effect of group, $F(1,24)=2.646$, $\mathrm{MSE}=2472.600, \mathrm{p}=0.117$. However, the lower extremity muscle strength of the training group was higher than that of the control group at the 12 th week of training.

\section{DISCUSSION}

The main finding of this study is that a home-based walking exercise increased the lower extremity muscle strength of hemodialysis patients. A decline in skeletal muscle strength is the main factor responsible for the reduction of exercise tolerance in hemodialysis patients ${ }^{20)}$ as

Table 2. Percent change of weekly walking data during 12 weeks of training in training group

\begin{tabular}{lcc}
\hline \multirow{2}{*}{ Week } & \multicolumn{2}{c}{ Percent change of number of steps/day } \\
\cline { 2 - 3 } & Non-HD & HD \\
\hline Pre & 100 & 100 \\
$1-2$ & $128.91 \pm 14.63$ & $144.83 \pm 50.47$ \\
$3-4$ & $155.13 \pm 22.86$ & $149.98 \pm 65.54$ \\
$5-6$ & $170.49 \pm 36.25$ & $173.94 \pm 79.84$ \\
$7-8$ & $196.79 \pm 43.91$ & $169.31 \pm 68.10$ \\
$9-10$ & $213.03 \pm 58.89$ & $207.76 \pm 111.48$ \\
$11-12$ & $237.82 \pm 75.34$ & $206.84 \pm 132.91$ \\
\hline
\end{tabular}

Data represent percent change from pre-study data. Non-HD $=$ non hemodialysis day; $\mathrm{HD}=$ hemodialysis day. shown by their low daily physical activity and low exercise capacity $^{21)}$. Tudor-Locke and Bassett ${ }^{22)}$ classified physical activity measured by a pedometer into four levels: sedentary, at $<5,000 \mathrm{steps} /$ day, inactive, at 5,000-7,499 steps/day, somewhat active, at 7,500-9,999 steps/day, and active, at $\geq 10,000$ steps/day. The present study data indicate that the patients had "sedentary" and "inactive" levels of physical activity on dialysis and non-dialysis days, respectively. Therefore, the baseline daily walking data of the training group showed that these patients had a low physical activity level and were in the chronic disease population. Individual goal setting is more effective in clinical practice than universal goals. Thus, in the present study, the individual exercise program prescribed to each patient depended on the baseline capacity of that patient. The total amount of physical activity in the present study was set at $110 \%$ of a patient's average number of steps at the baseline and gradually increased. Exercise intensity increased at the rate of $10 \%$ every two weeks. After participating in the study for 12 weeks, the patients showed an increase in their daily number of steps. According to the Tudor-Locke and Bassett's categories ${ }^{22)}$, the patients' activity levels improved to "somewhat active" and "active" on dialysis and non-dialysis days, respectively. Thus, this walking program increased the patients' levels of physical activity on both hemodialysis and non-dialysis days. The results of the present study show that the lower extremity muscle strength of the patients was lower than that of healthy subjects ${ }^{24)}$. The training group subjects showed a $31 \%$ increase in lower extremity muscle strength after twelve weeks of walking exercise. In contrast, lower extremity muscle strength did not change in the control group. Moreover, this walking program increased muscle strength after eight weeks of training. A previous investigation ${ }^{24)}$ indicated that low intensity exercise, such as walking, may provide adequate resistance to improve the muscle function of hemodialysis patients. The exact mechanisms explaining the improvement in skeletal muscle function and exercise capacity in hemodialysis patients are not clear. However, Kouidi et al. ${ }^{6)}$ reported the effects of combined aerobic and strength training for six months on the muscles of hemodialysis patients. Their training rehabilitation program consisted of a much more intense and varied exercise regime (10 min cycle ergometry or treadmill warm-up, followed by $50 \mathrm{~min}$ of either aerobic, exercise, swimming or ball games, $10 \mathrm{~min}$ of low-weight resistant exercise, $10 \mathrm{~min}$ of stretching and $10 \mathrm{~min}$ of cool-down). They found that the cross-sectional area of type I and II muscle fibers significantly increased. Moreover, ultrastructural analysis revealed that the muscles appeared to be normal, including positive adaptations of capillaries and mitochondria ${ }^{6}$. They suggested that this change was primarily related to the effects of aerobic training on enhanced oxidative capacity, and other muscle metabolism and functional energy by exercise training. Moreover, individuals with deconditioning states exhibit large initial gains in muscle function because of a large adaptation potential ${ }^{25}$. However, determining the type of walking exercises in the present study as either aerobic or 
strengthening exercise is difficult. The exercise prescription given to each patient consisted of a target number of steps to be accumulated by each patient over the course of a nondialysis day for at least three days per week. No further instructions related to daily walking exercise were given, such as recommendations for speed or duration. The patients were only instructed to accumulate steps until their targets were reached. There were limitations to the present study. First, the sample size was small, and replication using a larger number of subjects is recommended. Second, there was no data for daily numbers of steps in the control group throughout the experiment. A comparison of the number of steps in both the training and control groups before and after participation in the experiment is recommended. However, hemodialysis patients can more easily comply with this program than with aerobic or strengthening exercise programs. Moreover, all of the patients who participated in this study completed the program without dropping out. The home-based walking exercise program required only a pedometer and daily walking exercise. This result indicates that a home-based walking exercise program is suitable for hemodialysis patients whose daily physical activity has declined to a sedentary state. Given the results of this study, a walking exercise program could be developed into a daily self-managed exercise program, if instructions regarding the appropriate number of steps are prescribed.

\section{REFERENCES}

1) Johansen KL, Chertow GM, Ng AV, et al.: Physical activity levels in patients on hemodialysis and healthy sedentary controls. Kidney Int, 2000, 57: 25642570 .

2) DeOreo P: Hemodialysis patient-assessed functional health status predicts continued survival, hospitalization, and dialysis-attendance compliance. Am J Kidney Dis, 1997, 30: 204-212.

3) Churchill D, Torrance G, Taylor D: Measurement of quality of life in endstage renal disease: The time trade-off approach. Clin Invest Med, 1987, 10: 14-20.

4) Johansen KL, Shubert T, Douyle J, et al.: Muscle atrophy in patients receiving hemodialysis: Effects on muscle strength, muscle quality, and physical function. Kidney Int, 2003, 63: 291-297.

5) Oh-Park M, Fast A, Gopal S, et al.: Exercise for dialyzed: Aerobic and strength training during hemodialysis. Am J Phys Med Rehabil, 2002, 81: $814-821$.

6) Kouidi E, Albani M, Natsis K, et al.: The effects of exercise training on muscle atrophy in haemodialysis patients. Nephrol Dial Transplant, 1998, 13: 685699.

7) DePaul V, Moreland J, Eager T, et al.: The effectiveness of aerobic and muscle strength training in patients receiving hemodialysis and EPO: A randomized controlled trial. Am J Kidney Dis, 2002, 40: 1219-1229.

8) Konstantinidou E, Koukouvou G, Kouidi E, et al.: Exercise training in patients with end-stage renal disease on hemodialysis: comparison of three rehabilitation programs. J Rehabil Med, 2002, 34: 40-45.

9) Pate RR, Pratt M, Blair SN, et al.: Physical activity and public health: a recommendation from the centers for disease control and prevention and the american college of sports medicine. JAMA, 1995, 273: 402-407.

10) Marcus B, King T, Clark M, et al.: Theories and techniques for promoting physical activity behaviors. Sports Med, 1996, 22: 321-331.

11) Malagoni AM, Catizone L, Mandini S, et al.: Acute and long-term effects of an exercise program for dialysis patients prescribed in hospital and performed at home. J Nephrol, 2008, 21: 871-878.

12) Walsh J, Charlesworth A, Andrews R, et al.: Relation of daily activity levels in patients with chronic heart failure to long term prognosis. Am J Cardilo, 1997, 79: 1364-1369.

13) Schonhofer B, Ardes P, Geibel M, et al.: Evaluation of a movement detector to measure daily activity in patients with chronic lung disease. Eur Respir J, 1997, 10: 2814-2819.

14) Schmalzried TP, Szuszczewicz ES, Northfield MR, et al.: Quantitative assessment of walking activity after total hip or knee replacement. J Bone And Joint Surgery Am, 1998, 80-A: 54-59.

15) Bassey EJ, Dallosso HM, Fentem PH, et al.: Validation of a simple walking mechanical accelerometer (pedometer) for the estimation of walking activity. Eur J Appl Physiol, 1997, 56: 323-330.

16) American Thoracic Society: ATS statement: Guidelines for the six-minute walk test. Am J Respir Crit Care Med, 2002, 166: 111-117.

17) Coldwells A, Atkinson G, Reilly T: Sources of variation in back and leg dynamometry. Ergonomics, 1994, 73: 79-86.

18) Heyward V: Advanced fitness assessment exercise prescription (3rd ed). Champaign: Human Kinetics, 1998.

19) Santiworakul A, Jalayondeja W, Chantarothorn S, et al.: Effects of lower extremity exercise on muscle strength and physical capacity in COPD patients. J Med Assoc Thai, 2009, 92: 556-563.

20) Diesel W, Emms M, Knight BK, et al.: Morphologic features of myopathy associated with chronic renal failure. Am J Kidney Dis, 1993, 22: 667-684.

21) Endo F, Asakawa $Y$, Usada $S$, et al.: Effects of daily walking exercise on chronic hemodialysis outpatients. J Phys Ther Sci, 1996, 8: 1-4.

22) Tudor-Locke C, Bassett D: How many steps/day are enough?: preliminary pedometer indices for public health. Sports Med, 2004, 34: 1-8.

23) Sport Authority of Thailand: Physical fitness norms of Thai population. Bangkok: Sports Science Sector, Sport Science Department, Sports Authority of Thailand, 2000.

24) Storer T, Casaburi R, Sawelson S, et al.: Endurance exercise training during haemodialysis improves strength, power, fatigability and physical performance in maintenance haemodialysis patients. Nephrol Dial Transplant, 2005, 20: 1429-1437.

25) Fleck SL, Kraemer WJ: Designing resistance training programs. Champaign: Human Kinetics, 1997. 